\title{
Replacing animal use in teaching in Eastern European universities - an update
}

Belgrade, Serbia, 7-8 ${ }^{\text {th }}$ October 2009

The aims of the workshop were to explore ways in which the successful introduction of alternatives (computer-based and other) into the curricula could significantly reduce the number of animals used in teaching physiology and pharmacology in Eastern and Central European universities. A brief report of this workshop was written by Goran Krummenacher of the Doerenkamp-Zbinden Foundation and published in a recent volume of ALTEX (4/2009). Here we present a brief synopsis of the workshop findings and summary of the discussions and outcomes.

There were over 20 participants from 14 universities in 13 Eastern European countries. All were either physiologists or pharmacologists, teaching a range of undergraduate and postgraduate courses such as medicine, pharmacy, biological sciences, dentistry and nursing.

Before the workshop they were requested to complete a questionnaire which sought information about current animal use in teaching physiology and pharmacology at their universities and their perceptions of what might assist or inhibit the replacement of animal classes with alternatives. The survey was delivered online and as an MSWord document that could be completed and returned to the University of Edinburgh. The surveys were analysed and the outcomes presented during the workshop to stimulate discussion.

Eleven of the fourteen universities represented were using animals in teaching medicine, pharmacy, veterinary medicine, zoology and biology, with no animals being used by the workshop participants from Estonia, Lithuania, Poland and Serbia.
Universities in Romania, Turkey, Bulgaria and Macedonia were the largest users in this survey.

In descending order the major animal species still being used were rat, frog/toad, mouse, rabbit, and guinea pig. Three institutions reported using more than 100 animals per year in teaching. Most animals were used for experiments on the cardiovascular and neuromuscular systems.

When asked about what would persuade them to replace an existing animal experiment with an alternative, the main responses were: students objecting to using animals in teaching; recommendation from a colleague; lower cost of using an alternative; evidence of educational effectiveness and positive independent reviews. In contrast the reasons they gave for not introducing alternatives were: lack of local language versions of alternatives; (high) cost of alternatives and lack of computer facilities.

It was clear from this admittedly very small sample that animal use in teaching physiology and pharmacology in Eastern European universities is still significant and that there is potential to reduce it. Many of the animal preparations used for teaching were similar to those used in universities in Western Europe for which, in many instances, (computer-based) alternatives already exist and offer direct replacements. However, in some cases, animal experiments for which there are no suitable alternatives were also used in teaching.

Participants expressed a willingness to consider alternative methods, though a serious concern was that the vast majority of the alternatives available were written in English and, 
while it was recognised that this is the global scientific language, the view was that having only English versions available does impede implementation. Students prefer to learn in their native language and faculty are more comfortable in using native language teaching resources. Many of the participants felt that animal use would continue, even if there were good (computer-based) alternatives available, because of this language barrier.

As a consequence it was agreed that the newly-formed consortium would apply for grant funding to enable the introduction of at least one local language version of a computer-based alternative in each university*. Faculty from each university would identify an animal class for which there was an existing (English language) replacement alternative available. They would then work with the University of Edinburgh to translate an existing English computer program into their native language. This new version would be made freely available to other universities in the consortium and beyond. The faculty would commit to using the new computer program as a replacement for the animal lab in the next academic session and carry out an evaluation seeking the views of both students and faculty. It was agreed that as far as possible the evaluation should be standardised and that a robust, simple-to-apply methodology, which would deliver useful data, would need to be developed.

Professor Dr. David Dewhurst

College of Medicine \& Veterinary Medicine

University of Edinburgh, Hugh Robson Building

15 George Square

Edinburgh EH8 9XD, UK

e-mail: d.dewhurst@ed.ac.uk

Professor Dr. Zvezdana Z. Kojic, MD, PhD

University of Belgrade

School of Medicine, Insitute of Physiology

Visegradska 26

11000 Belgrade, Serbia

e-mail: zvezdanak@med.bg.ac.rs

\footnotetext{
* A funding proposal to continue this work was submitted to DZF in October 2009 and has been successful. Work has already started on the development of local language versions of certain computer-based alternatives and these will be implemented in the next academic year. We hope to report the results of this project in 2011.
} 